\title{
Hydrocortisone Inhibits Cellular Proliferation by Downregulating Hepatocyte Growth Factor Synthesis in Human Osteoblasts
}

\author{
Yoshihiko Tsunashima, Ayami Kondo, ${ }^{\dagger}$ Tomohiro Matsuda, and Akifumi Togari* \\ Department of Pharmacology, School of Dentistry, Aichi-Gakuin University; Nagoya 464-8650, Japan. \\ Received January 7, 2011; accepted February 17, 2011; published online February 21, 2011
}

\begin{abstract}
Glucocorticoids have multiple systemic effects that may influence bone metabolism but also directly affect osteoblasts by decreasing their proliferation. Using human osteoblastic SaM-1 cells, we examined whether the effects of hydrocortisone on cellular proliferation are mediated by hepatocyte growth factor (HGF). Human osteoblasts constitutively express both HGF and c-Met, its receptor. Hydrocortisone decreased the gene and protein expression of HGF as well as proliferation in SaM-1 cells. These hydrocortisone ( $\left.0.01-1 \mu_{\mathrm{M}}\right)$-induced decreases in HGF synthesis and cellular proliferation occurred in a concentration-dependent manner. However, no hydrocortisone $\left(0.01-1 \mu_{\mathrm{M}}\right)$-induced decrease in cellular proliferation was observed in human osteosarcoma-derived cells (HOS and SaOS-2), which are not able to produce HGF. In the cellular proliferation in SaM-1 cells, the decrease was blocked concentration-dependently by exogenously applied HGF $(0.01-3 \mathrm{ng} / \mathrm{ml})$. Furthermore,

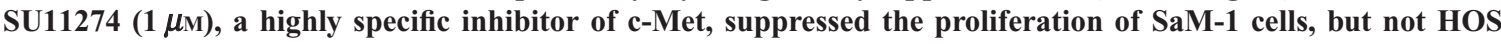
cells. From these results, we concluded that hydrocortisone inhibits the proliferation of SaM-1 cells by interrupting the autocrine/paracrine loop via the downregulation of HGF synthesis.
\end{abstract}

Key words osteoblast; hydrocortisone; hepatocyte growth factor; proliferation

Glucocorticoids impair the proliferation, differentiation, and function of osteoblasts and induce apoptosis in mature osteoblasts. ${ }^{1-6)}$ In addition to their direct effects on osteoblasts, they have also been demonstrated to modify the synthesis of growth factors produced by osteoblastic cells. ${ }^{7-9)}$ These effects lead to the suppression of bone formation, a central feature in the pathogenesis of glucocorticoid induced osteoporosis. Hepatocyte growth factor (HGF), which was initially thought to be of mesodermal origin, and its receptor are expressed in osteoblasts and osteoclasts. ${ }^{10-12)}$ Previous studies have shown the inhibitory effects of glucocorticoids on HGF mRNA expression in rat calvarial osteoblasts ${ }^{13)}$ and human osteoblast-like cells, ${ }^{14)}$ and the stimulatory effects of HGF on thymidine incorporation in osteoblasts. ${ }^{11)}$ These findings led us to investigate whether the effects of glucocorticoids on cellular proliferation are mediated by HGF in human osteoblastic cells. In this study, using osteoblastic cells (SaM-1) and osteogenic sarcoma cells (SaOS-2, HOS, and MG-63), we demonstrate that HGF functions in an autocrine and/or paracrine manner, resulting in mitogenesis. Furthermore, we show that the inhibitory effects of glucocorticoid on the proliferation of human osteoblastic cells can probably be explained by the concomitant glucocorticoidinduced inhibition of HGF production.

\section{MATERIALS AND METHODS}

Materials Hydrocortisone was obtained from Sigma (St. Louis, MO, U.S.A.), and recombinant human HGF protein and a human HGF enzyme-linked immunosorbent assay (ELISA) system were obtained from R\&D systems (Minneapolis, MN, U.S.A.). The bromodeoxyuridine (BrdU) cell proliferation ELISA was from Roche (Penzberg, Germany). QIAzol lysis reagent was obtained from Qiagen (Hilden, Germany). SU11274 [(3Z)- $N$-(3-chlorophenyl)-3-(\{3,5-di-

\footnotetext{
${ }^{\dagger}$ Present address: Department of Medicinal Biochemistry, School of

Pharmacy, Aichi-Gakuin University; Nagoya 464-8650, Japan.
}

methyl-4-[(4-methylpiperazin-1-yl)carbonyl]-1H-pyrrol-2yl \}methylene)- $N$-methyl-2-oxo-2,3-dihydro- $1 H$-indole-5-sulfonamide] was obtained from CalBiochem (San Diego, CA, U.S.A.). Normal human osteoblasts (SaM-1 cells) were kindly provided by Dr. Koshihara (Tokyo Metropolitan Institute of Gerontology), who prepared them from an explant of ulnar periosteum obtained from a 20 -year-old male patient undergoing curative surgery who gave his informed consent for its experimental use. SaM-1 cells have a mitotic life span of 34 population doubling levels (PDL), ${ }^{15)}$ and we used them at 23-24 PDL in our experiments. HOS and SaOS-2 human osteogenic sarcoma cells were obtained from the RIKEN Cell Bank (Tsukuba, Japan). MG-63 human osteogenic sarcoma cells were from the American Type Culture Collection (Rockville, MD, U.S.A.). Alpha-modified minimum essential medium ( $\alpha$-MEM) was purchased from Gibco BRL (Grand Island, NY, U.S.A.), and fetal calf serum (FCS) was obtained from Cell Culture Laboratories (Cleveland, OH, U.S.A.) and Irvine Scientific (Santa Ana, California, U.S.A.). All other chemicals used were of reagent grade.

Cell Culture The medium used for all cell lines was $\alpha$ MEM containing $10 \%$ fetal calf serum (FCS), and the cells were incubated in a humidified incubator at $37{ }^{\circ} \mathrm{C}$ in $95 \%$ air and $5 \% \mathrm{CO}_{2}$. In an analysis of proliferation, SaM-1 cells were seeded in 96-well plates at a density of $3 \times 10^{3}$ cells/well and allowed to settle for $24 \mathrm{~h}$, and then the medium was replaced with $\alpha$-MEM containing $2.5 \%$ FCS and hydrocortisone. All osteogenic sarcoma cells were seeded in 96-well plates at a density of $2 \times 10^{3}$ cells/well and allowed to settle for $24 \mathrm{~h}$, and then the medium was replaced with $\alpha$-MEM (serum free) containing hydrocortisone. For the analysis of gene expression, SaM-1 cells were cultured in $6 \mathrm{~cm}$ dishes at a density of $1.5 \times 10^{5}$ cells/dish (proliferative stage) for the indicated periods, and then the total RNA was extracted using QIAzol Lysis reagent. The other cell lines were cultured in 10 -cm dishes at a density of $1 \times 10^{5}$ cells/dish (proliferative stage) for the indicated periods, and then the total RNA was extracted. 
Cellular Proliferation Assay After treatment with hydrocortisone for $24 \mathrm{~h}$, BrdU was added to each well to a final concentration of $10 \mu \mathrm{M}$, and the cells were incubated for $4 \mathrm{~h}$ (SaM-1 and SaOS-2 cells) or $1 \mathrm{~h}$ (HOS and MG-63 cells). The amount of BrdU-labeled DNA was quantified using the ELISA system, and the data were expressed as a percentage of the control, which was taken as $100 \%$.

Reverse Transcription-Polymerase Chain Reaction (RT-PCR) Analysis Total RNA were treated with DNase I (Boehringer Mannheim, GmbH, Germany), and cDNA was synthesized using random primers and Moloney murine leukemia virus reverse transcriptase (Gibco-BRL, Grand Island, NY, U.S.A.), which was followed by PCR amplification using synthetic gene primers specific for human HGF, c-Met, and human glyceraldehyde 3-phosphate dehydrogenase (GAPDH) as a housekeeping gene. These primers were designed with reference to the reported cDNA sequences. ${ }^{16-18)}$ The oligonucleotide primers were synthesized on a DNA synthesizer (Expedite model 8909; PerSeptiv Biosystem, Cambridge, MA, U.S.A.) and purified using a polypropylene filter (Oligo Prep kit; Pharmacia Biotech, Uppsala, Sweden). The forward and reverse primers for the genes examined and the sizes of the amplified product were as follows: GAPDH, 5'-ACCACAGTCCATGCCATCAC-3' and 5' -TCCACCACCCTGTTGCTGTA-3', 452-bp cDNA fragment; HGF, 5'ATTGCCCTATTTCTCGTTGTG-3' and 5'-GCATTTCTCATCTCCTCTTCC-3', 296-bp cDNA fragment; c-Met, 5' CTGGGAGCTGATGACAAGAGG-3' and 5'-TGACAACAGAGAAGGATACGG-3', 292-bp cDNA fragment. PCR amplification was performed with a GeneAmp PCR System (Perkin Elmer/Cetus, Norwalk, CT, U.S.A.) according to the following settings: denaturation at $95^{\circ} \mathrm{C}$ for $15 \mathrm{~s}$, annealing at $55^{\circ} \mathrm{C}$ for $30 \mathrm{~s}$, and elongation at $72{ }^{\circ} \mathrm{C}$ for $30 \mathrm{~s}$ for the appropriate number of cycles. The PCR products were electrophoresed on a $2 \%$ Nusieve GTG agarose gel (FMC BioProducts, Rockland, ME, U.S.A.), stained with ethidium bromide, and detected with a fluoroimage analyzer (FluorImager 575; Molecular Dynamics, Sunnyvale, CA, U.S.A.).

Measurement of HGF Production The amount of HGF in conditioned media was quantified using ELISA kits. After treatment with hydrocortisone for $3,6,24$, or $48 \mathrm{~h}$, conditioned medium was used for ELISA analysis. Data are presented as the mean \pm S.E.M. of 3 cultures.

\section{RESULTS}

Effects of Hydrocortisone on the Expression of HGF and c-Met in SaM-1 Cells The effects of hydrocortisone on HGF expression in SaM-1 cells were assessed by RT-PCR and ELISA analysis. Figure 1A shows the time-course of the effects of hydrocortisone on the expression of HGF and cMet mRNA in SaM-1 cells. Substantial amounts of these mRNAs were detected under steady-state conditions used, but treatment of the cells with hydrocortisone $(1 \mu \mathrm{M})$ rapidly reduced HGF mRNA expression without affecting the expression of HGF receptor; i.e., c-Met mRNA. The reduction in the mRNA expression of HGF was largest at $6 \mathrm{~h}$ and had slightly declined after $24 \mathrm{~h}$ of treatment. In addition, we also confirmed that the SaM-1 cells produced HGF protein. As shown in Fig. 1B, HGF accumulated in the SaM-1 cell culture medium, in a time-dependent manner. The production of
A
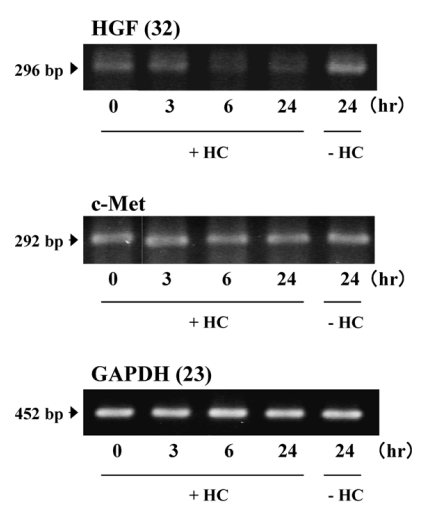

B

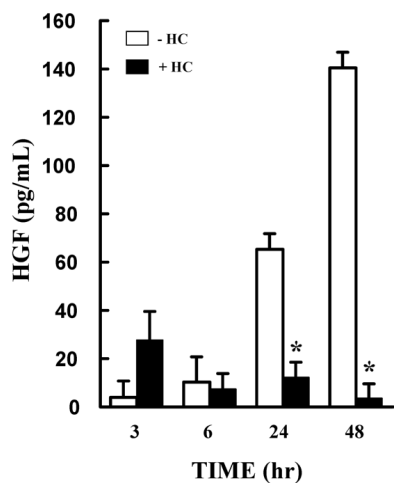

Fig. 1. Effects of Hydrocortisone on the Expression of HGF and c-Met in SaM-1 Cells

(A) Gene expression levels of HGF and c-Met in SaM-1 cells were determined by RT-PCR analysis. mRNA were extracted from cells incubated in the absence or presence of hydrocortisone (HC; $1 \mu \mathrm{M}$ ) for 3,6 , or $24 \mathrm{~h}$. GAPDH was used as housekeeping gene. The numbers in parentheses indicate cycles of PCR amplification. The arrow indicates the predicted size of the PCR product. The results obtained using a specific primer for GAPDH are shown for comparison. (B) HGF protein expression in SaM-1 was determined using an ELISA system. HGF secreted into conditioned medium from cells incubated in the absence (opens) or presence (solids) of hydrocortisone $(1 \mu \mathrm{M})$ for $3,6,24$, or 48 h. $* p<0.01 v$ s. non treated cells (Student $t$-test; $n=3$ ).
A

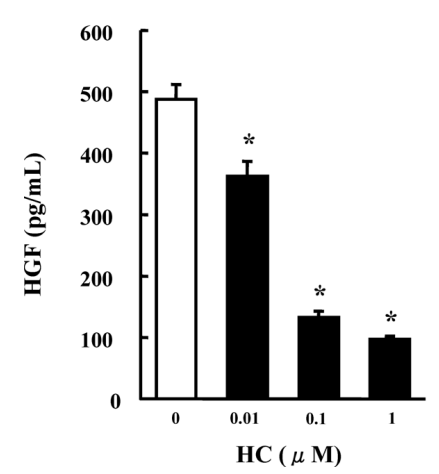

B

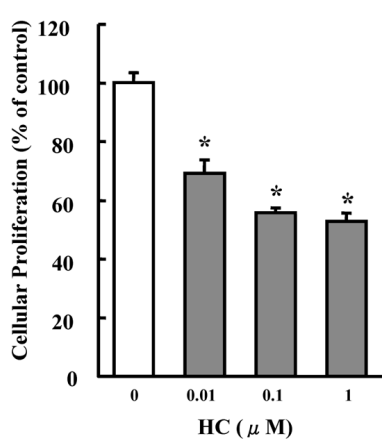

Fig. 2. Dose-Dependent Effects of Hydrocortisone on HGF Expression (A) and Cellular Proliferation (B) in SaM-1 Cells

(A) Cells were treated with different concentrations of hydrocortisone (HC) for $24 \mathrm{~h}$, and the cell culture medium served as a sample for determining HGF production using an ELISA system. $* p<0.01 v s$. non-treated cells (ANOVA and post-hoc Bonferroni; $n=3$ ). (B) Cells were treated with different concentrations of hydrocortisone (HC) for $24 \mathrm{~h}$ and then were incubated with BrdU for $4 \mathrm{~h}$. A cellular proliferation assay was performed to detect BrdU-labeled DNA using anti-BrdU monoclonal antibody labeled with peroxidase and expressed as a percentage of BrdU incorporation in untreated cells $* p<0.01 v s$. non-treated cells (ANOVA and post-hoc Bonferroni; $n=5$ ).

HGF was significantly reduced by treatment with hydrocortisone $(1 \mu \mathrm{M})$.

Dose-Dependent Effects of Hydrocortisone on HGF Expression and Cellular Proliferation in SaM-1 Cells To analyze the cellular responses to hydrocortisone, we examined its effects on cellular proliferation by measuring BrdU incorporation. Figure 2 shows the concentration-dependent effects of hydrocortisone on HGF expression and cellular proliferation in SaM-1 cells. Both HGF synthesis and cellular proliferation were reduced by treatment with hydrocortisone for $24 \mathrm{~h}$, and these reductions were concentrationdependent in the range between $0.01-0.1 \mu \mathrm{M}$. In addition, hydrocortisone had an inhibitory effect on cellular proliferation after treatment for $48 \mathrm{~h}$ (data not shown). 


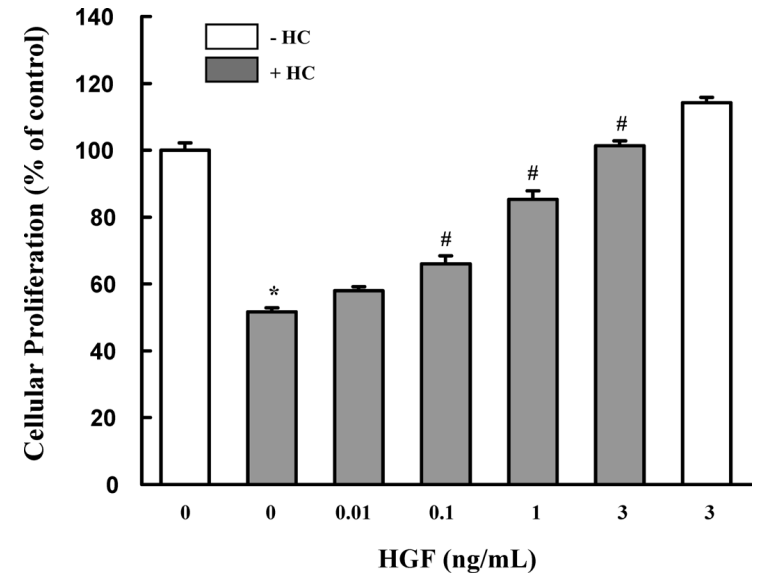

Fig. 3. Effects of Exogenously Applied HGF on the Suppression of SaM1 Cell Proliferation by Hydrocortisone

The cells were treated with hydrocortisone $\left(\mathrm{HC} ; 10^{-6} \mathrm{M}\right)$ for $24 \mathrm{~h}$ and then were incubated with BrdU for $4 \mathrm{~h}$. To examine the effects of HGF, different concentrations of HGF were added to cells that had been treated with hydrocortisone for $6 \mathrm{~h}$. A cellular proliferation assay was performed to detect BrdU-labeled DNA usung anti-BrdU monoclonal antibody labeled with peroxidase and expressed as a percentage of BrdU incorporation in non-treated cells (control). $* p<0.01 v s$. non-treated cells, $\# p<0.01 v s$. HC treated cells (ANOVA and post-hoc Bonferroni; $n=5$ ).

Effects of Exogenously Applied HGF on the Suppression of SaM-1 Cell Proliferation by Hydrocortisone To elucidate whether the effects of hydrocortisone on cellular proliferation are mediated by HGF, we examined the influence of exogenously applied HGF on the effects of hydrocortisone on cellular proliferation in SaM-1 cells. As shown in Fig. 3, the inhibitory effect of hydrocortisone on cellular proliferation was suppressed by treatment with exogenous HGF. The effects of exogenous HGF were concentration-dependent in the range between $0.01-3 \mathrm{ng} / \mathrm{ml}$, and treatment of the cells with $3 \mathrm{ng} / \mathrm{ml} \mathrm{HGF}$ recovered cellular proliferation to the control level.

Effects of Hydrocortisone on HGF Expression and Cellular Proliferation in Human Osteogenic Sarcoma Cells A similar experiment was performed in human osteogenic sarcoma cells, SaOS-2, HOS, and MG-63 cells. Table 1 shows the effects of hydrocortisone treatment for $24 \mathrm{~h}$ on HGF synthesis and cellular proliferation in these cells. Hydrocortisone had no influence on cellular proliferation in SaOS-2 or HOS cells, but inhibited it in a concentrationdependent manner in MG-63 cells. In addition, using the ELISA system, we could not detect HGF in SaOS-2 or HOS cell conditioned medium in the absence or presence of hydrocortisone. However, HGF synthesis was detected under steady-state conditions in MG-63 cells, and their proliferation was significantly reduced by hydrocortisone $\left(10^{-7}\right.$ $\left.10^{-6} \mathrm{M}\right)$. Thus, in SaM-1 as well as MG-63 cells, hydrocortisone had an inhibitory effect on cellular proliferation and HGF synthesis. However, in SaOS-2 and HOS cells, which do not express HGF under steady-state conditions, hydrocortisone did not reduce cellular proliferation. On the other hand, osteogenic sarcoma cells (MG-63, SaOS-2, and HOS) were able to express c-Met mRNA, substantially (data not shown).

Effects of SU11274 on the Cellular Proliferation of SaM-1 and HOS Cells Next, to elucidate the involvement of autocrine/paracrine systems in the effects of HGF on cel-
Table 1. Effects of Hydrocortisone on HGF Expression and Cellular Proliferation in Human Osteogenic Sarcoma Cells

\begin{tabular}{clcc}
\hline \hline Cell & $\begin{array}{c}\text { Hydrocortisone } \\
(\mu \mathrm{M})\end{array}$ & $\begin{array}{c}\text { Proliferation } \\
(\% \text { of control })\end{array}$ & $\begin{array}{c}\text { HGF } \\
(\mathrm{pg} / \mathrm{ml})\end{array}$ \\
\hline \multirow{2}{*}{ SaOS-2 } & 0 (control) & $100.0 \pm 8.4$ & n.d. \\
& 0.01 & $103.3 \pm 0.4$ & n.d. \\
& 0.1 & $98.6 \pm 12.7$ & n.d. \\
HOS & 1 & $106.1 \pm 2.9$ & n.d. \\
& 0 (control) & $100.0 \pm 2.6$ & n.d. \\
& 0.01 & $107.3 \pm 3.7$ & n.d. \\
MG-63 & 0.1 & $94.6 \pm 2.8$ & n.d. \\
& 0 (control) & $108.4 \pm 2.7$ & n.d. \\
& 0.01 & $93.3 \pm 4.5$ & $676.5 \pm 33.5$ \\
& 0.1 & $75.9 \pm 5.7^{*}$ & $305.8 \pm 8.2^{*}$ \\
& 1 & $64.2 \pm 1.3^{*}$ & $320.3 \pm 27.0^{*}$ \\
& & &
\end{tabular}

SaOS-2, HOS, and MG-63 cells were treated with different concentrations of hydrocortisone for $24 \mathrm{~h}$, and then the HGF concentration in cultured medium and cellular proliferation were determined. $* p<0.01 \mathrm{vs}$. non-treated (control) cells (ANOVA and post-hoc Bonferroni; $n=5$ ). n.d.: not detected.
A

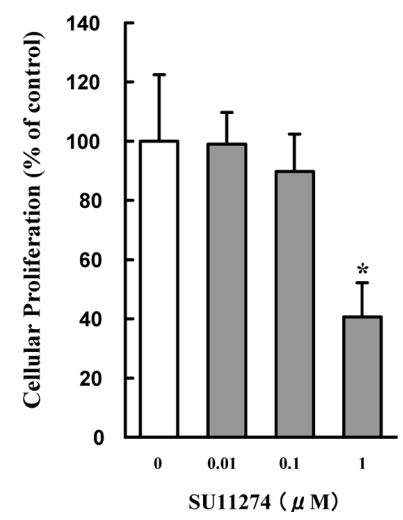

B

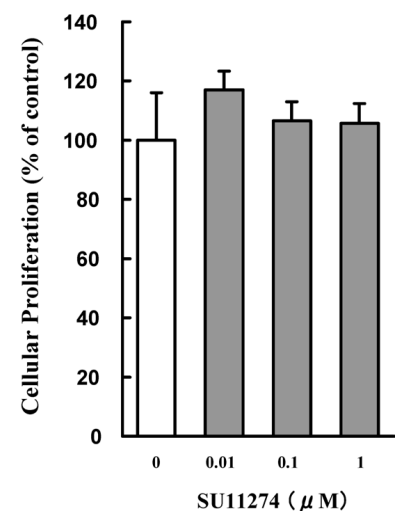

Fig. 4. Effect of SU11274 on Cellular Proliferation in SaM-1 (A) and HOS (B) Cells

SaM-1 and HOS cells were treated with different concentrations of SU11274 for $24 \mathrm{~h}$ and then were incubated with BrdU for $4 \mathrm{~h}$ (SaM-1 cells) or $1 \mathrm{~h}$ (HOS cells). A cellular proliferation assay was performed to detect BrdU-labeled DNA using anti-BrdU monoclonal antibody labeled with peroxidase and expressed as a percentage of BrdU incorporation in untreated cells. $* p<0.01 v s$. non-treated cells (ANOVA and post-hoc Bonferroni; $n=5$ ).

lular proliferation in osteoblastic cells, we examined the effects of SU11274, which is a highly specific inhibitor of cMet, on cellular proliferation in the various osteoblastic cell lines examined in this study. As shown in Fig. 4, the proliferation of SaM-1, but not HOS, cells was decreased by treatment with SU11274 $(1 \mu \mathrm{M})$. These show the cellular regulation of endogenously secreted HGF on proliferation of SaM1 cells.

\section{DISCUSSION}

Glucocorticoids have marked and complex effects on bone formation and resorption, although their inhibitory effects on bone formation appear central to the bone loss observed after glucocorticoid treatment. ${ }^{19,20)}$ Glucocorticoids decrease the pool of available osteoblasts through apoptosis. ${ }^{21)}$ In addition, they have direct effects on specific genes expressed by osteoblasts and regulate the synthesis of locally produced growth factors. ${ }^{7,8)}$ Hepatocyte growth factor (HGF) is ex- 
pressed almost ubiquitously, including in osteoblasts ${ }^{10,12)}$ and osteoclasts, ${ }^{11)}$ and exerts autocrine and/or paracrine effects depending on the distribution of its receptor, the protooncogene c-Met, which is a tyrosine kinase-activated receptor. ${ }^{22)}$ This receptor is also expressed in normal human osteoblasts and osteoclasts ${ }^{11,13)}$ as well as various tumors of skeletal origin. ${ }^{23,24)}$ In the present study, the constitutive expression of HGF and c-Met was identified in human osteoblastic SaM-1 cells. Hydrocortisone not only causes decreased cellular proliferation, but also inhibits the expression of HGF, without affecting c-Met expression in cultures of SaM-1 cells. This prompted us to explore whether HGF acts as a mediator of the effects of glucocorticoids on cellular proliferation in human osteoblasts.

To elucidate whether the effects of hydrocortisone on cellular proliferation are mediated by HGF, the influence of exogenous HGF on the effects of hydrocortisone on SaM-1 cell proliferation was examined. As shown in the present study, the inhibitory effect of hydrocortisone on cellular proliferation was recovered by treatment with exogenous HGF. At the concentration used in the present study, it has been reported to induce biological responses both in osteoclasts and in osteoblasts. ${ }^{11)}$ Thus, exogenous HGF antagonized the action of hydrocortisone on cellular proliferation in SaM-1 cells. Furthermore, hydrocortisone did not alter the cellular proliferation of osteogenic sarcoma cells, which do not produce HGF under basal conditions. These findings suggest that one of the mechanisms underlying the inhibition of SaM-1 proliferation by hydrocortisone is the inhibition of HGF synthesis.

A question has been raised as to whether an autocrine/ paracrine system for HGF is involved in SaM-1 cell proliferation, and the inhibitory effects of hydrocortisone on cellular proliferation may be based upon interruption of the autocrine/paracrine loop. This possibility was reinforced by the detection of the c-Met transcript in these cells, as assessed by RT-PCR. To investigate the functionality of this expressed HGF receptor, we tested the cellular response to a highly specific inhibitor of c-Met, ${ }^{25)}$ a receptor tyrosine kinase. To elicidate the involvement of the autocrine/paracrine system for HGF in osteoblastic cell proliferation, we examined the effect of SU11274, a novel small-molecule tyrosine kinase inhibitor, on osteoblastic cell proliferation. At the $\mathrm{IC}_{50}$ concentration for cell growth in human melanoma cell lines, ${ }^{26}$ ) SU11274 significantly inhibited SaM-1 cell proliferation, probably because of high endogenous secretion of HGF; however, it did not inhibit the proliferation of HOS cells. These findings suggest that the inhibition of cellular proliferation by hydrocortisone is based upon interruption of the autocrine/paracrine loop via the downregulation of HGF synthesis in human osteoblasts, which produced a sufficient amount of HGF protein under control conditions.

In conclusion, we have demonstrated the existence of an autocrine/paracrine loop for HGF in human normal osteoblast, SaM-1 cells, that supports mitogenesis. Furthermore, hydrocortisone inhibits the proliferation of SaM-1 cells by interfering with this autocrine/paracrine loop by inhibiting HGF synthesis without any accompanying change in the expression of its receptor, c-Met. Our results suggest a new mechanism underlying glucocorticoid-mediated growth inhibition of human osteoblasts.

Acknowledgements The excellent technical assistance of Shoko Imamura is gratefully acknowledged. This study was partly supported by a Grant-in-Aid for Scientific Research (20592193 to A.T.) from the Japan Society for the Promotion of Science and by a Grant-in-Aid from Strategic Research AGU-Platform Formation (2008-2012).

\section{REFERENCES}

1) Hulley P. A., Gordon F., Hough F. S., Endocrinology, 139, 2423 2431 (1998).

2) Ishida Y., Heersche J. N., J. Bone Miner. Res., 13, 1822-1826 (1998).

3) Manolagas S. C., Endocr. Rev., 21, 115-137 (2000).

4) Porter R. M., Huckle W. R., Goldstein A. S., J. Cell Biochem., 90, $13-22$ (2003).

5) Liu Y., Porta A., Peng X., Gengaro K., Cunningham E. B., Li H., Dominguez L. A., Bellido T., Christakos S., J. Bone Miner. Res., 19, $479-490$ (2004).

6) Li X., Jin L., Cui Q., Wang G. J., Balian G., Osteoporos. Int., 16, $101-108$ (2005).

7) Canalis E., Centrella M., Burch W., McCarthy T. L., J. Clin. Invest., 83, 60-65 (1989).

8) Delany A. M., Canalis E., Endocrinology, 136, 4776- 4781 (1995).

9) Luppen C. A., Smith E., Spevak L., Boskey A. L., Frenkel B., J. Bone Miner: Res., 18, 1186-1197 (2003).

10) Blanquaert F., Delany A. M., Canalis E., Endocrinology, 140, 1069 1074 (1999).

11) Grano M., Galimi F., Zambonin G., Colucci S., Cottone E., Zallone A. Z., Comoglio P. M., Proc. Natl. Acad. Sci. U.S.A., 93, 7644-7648 (1996).

12) Taichman R., Reilly M., Verma R., Ehrenman K., Emerson S., Br. J. Haematol., 112, 438-448 (2001).

13) Blanquaert F., Pereira R. C., Canalis E., Am. J. Physiol. Endocrinol. Metab., 278, E509-E515 (2000).

14) Skrtic S., Ohlsson C., Calcif. Tissue Int., 66, 108-112 (2000).

15) Koshihara Y., Kawamura M., Oda H., Higaki S., Biochem. Biophys. Res. Commun., 145, 651-657 (1987).

16) Arcari P., Martinelli R., Salvatore F., Nucleic Acids Res., 12, 9179 9189 (1984).

17) Park M., Dean M., Kaul K., Braun M. J., Gonda M. A., Vande Woude G., Proc. Natl. Acad. Sci. U.S.A., 84, 6379-6383 (1987).

18) Miyazawa K., Tsubouchi H., Naka D., Takahashi K., Okigaki M., Arakaki N., Nakayama H., Hirono S., Sakiyama O., Takahashi K., Gohda E., Daikuhara Y., Kitamura N., Biochem. Biophys. Res. Commun., 163, 967-973 (1989).

19) Delany A. M., Dong Y., Canalis E., J. Cell Biochem., 56, 295-302 (1994).

20) Canalis E., Curr. Osteoporos. Rep., 3, 98-102 (2005).

21) Weinstein R. S., Jilka R. L., Parfitt A. M., Manolagas S. C., J. Clin. Invest., 102, 274-282 (1998).

22) Naldini L., Vigna E., Narsimhan R. P., Gaudino G., Zarnegar R., Michalopoulos G. K., Comoglio P. M., Oncogene, 6, 501-504 (1991).

23) Fukuda T., Ichimura E., Shinozaki T., Sano T., Kashiwabara K., Oyama T., Nakajima T., Nakamura T., Pathol. Int., 48, 757-762 (1998).

24) Ferracini R., Scotlandi K., Cagliero E., Acquarone F., Olivero M., Wunder J., Baldini N., J. Cell Physiol., 184, 191-196 (2000).

25) Matsumoto K., Nakamura T., Int. J. Cancer, 119, 477-483 (2006).

26) Puri N., Ahmed S., Janamanchi V., Tretiakova M., Zumba O., Krausz T., Jagadeeswaran R., Salgia R., Clin. Cancer Res., 13, 2246-2253 (2007). 